\title{
Vertebral Endplate Changes Correlate with Presence of Cartilaginous Endplate in the Herniated Disc Tissue: Factor Predicting Failure of Conservative Treatment
}

\author{
Rabia Latif ${ }^{1}$, Sumera Imran ${ }^{1}$, Ijaz Ahmad ${ }^{2}$, Muhammad Saad Ilyas ${ }^{2}$, Amer Aziz $^{2}$, Uruj Zehra ${ }^{1}$ \\ ${ }^{1}$ Department of Anatomy, University of Health Sciences Lahore, Lahore, Pakistan \\ ${ }^{2}$ Department of Orthopaedics \& Spine Surgery, Ghurki Trust Teaching Hospital, Lahore, Pakistan
}

Study Design: Cross-sectional comparative.

Purpose: To characterize the scores of disc degeneration, inflammation, and nerve density in herniated disc samples and associate findings with the presence of vertebral endplate (VEP) changes on magnetic resonance imaging (MRI).

Overview of Literature: Considering the role of disc composition in spontaneous regression and persistence of pain during conservative management, it is important to identify the influencing factors. VEP changes are highly associated with disc degeneration, but their correlation with herniated disc composition has not yet been reported.

Methods: Fifty-one discs were obtained from patients undergoing surgery for herniated disc. Their ages ranged from 19-65 years, and 31/51 were male. Pre-surgical T1 and T2 weighted lumbar-spine MRIs were analyzed to observe Pfirrmann grade, VEP defects, herniation type, Modic changes, and high-intensity zones (HIZ) at the affected level. Five-micron thick sections were stained with hematoxylin and eosin, Alcian blue periodic acid-Schiff stain; examined for histological degeneration scores (HDS; 0-15), inflammation (0 [absence]-3 [severe]), and presence of cartilaginous endplate (CEP). Three-micron thick sections were stained with protein-geneproduct 9.5 and expression was counted $/ \mathrm{mm}^{2}$. Data was analyzed, and $p<0.05$ was considered to indicate statistical significance.

Results: VEP defects, Modic changes, and HIZ were respectively observed in 30/51, 16/51, and 6/51 of the samples. CEP was observed in 26/51 samples and in 23/51 with endplate defects. Discs with adjacent VEP defects showed increased HDS $(p<0.001)$ and inflammation $(p<0.001)$. Discs with adjacent Modic changes also revealed increased HDS ( $p=0.01)$. Histological sections with CEP showed increased HDS $(p<0.001)$ and inflammation $(p<0.001)$, and nerve density was significantly positively correlated with HDS $(r=0.27, p=0.02)$.

Conclusions: VEP changes can modulate degeneration and inflammation of herniated discs. Presence of these changes is highly predictive of the occurrence of CEP in herniated discs, which leads to slow resorption and persistent clinical symptoms.

Keywords: Herniated discs; Intervertebral disc degeneration; Vertebral endplate

Received Mar 27, 2021; Revised May 1, 2021; Accepted May 2, 2021

Corresponding author: Uruj Zehra

Department of Anatomy, University of Health Sciences Lahore, Khayaban-e-Jamia Punjab, Lahore-54600, Pakistan

Tel: +92-3364785095, Fax:+92-042-99230870, E-mail: headanatomy@uhs.edu.pk 


\section{Introduction}

Intervertebral disc herniation refers to the displacement of disc material beyond the normal margins of the disc space and is a common form of disc degeneration [1]. Recurrent torsional strain may cause annular rupture, leading to extravasation of the disc material through the injured annulus. The herniated disc material usually includes elements of the nucleus pulposus, annulus fibrosus, or both [2].

The first line of treatment recommended for disc herniation is usually conservative unless the patient has severe neurological compromise; surgery is considered the last treatment option because it does not always yield predictable results [3]. The findings of observational studies on the outcomes of these two treatments show remarkable variability. While some studies conclude that surgical treatment provides faster pain relief in patients with lumbar disc herniation but fails to show distinct benefits over conservative treatment in mid-and long-term follow-up [4], other authors support surgical intervention for the rapid relief of symptoms and restoration of function [5]. As no objective biological markers with which to evaluate the severity of disc herniation other than pain and neurological symptoms are currently available [6], selecting an appropriate management strategy is often challenging.

The results of studies on the prognostic outcomes of herniated discs over the course of conservative treatment vary. Herniated discs, especially massively extruded discs, have a greater tendency to undergo regression with conservative management. The exact mechanism of disc regression remains unclear, but the size, integrity, and radiological and histological characteristics of the herniated discs are believed to play role in this process. The rate of spontaneous regression ranges from $13 \%-96 \%$ in various cases $[7,8]$, but the regression in size has no direct association with clinical improvement $[3,8,9]$. Researchers have pointed out that the presence of the cartilaginous endplate (CEP) in the herniated disc tissue may prolong the symptoms of a patient owing to the biological nature of the cartilage, which is slow to resorb $[10,11]$. The presence of CEP in the disc tissue could reflect vertebral endplate (VEP) damage at the same level, which may further modify the histological and histochemical features of herniated discs by increasing communication between the disc constituents and vascularized vertebral bone marrow. Although several lines of evidence indicate that VEP damage is associated with disc degeneration and lower back pain, how this damage affects the herniated disc tissue and pain-related characteristics remains unknown.

The current study aims to identify the factors influencing VEP defects on magnetic resonance imaging (MRI) and their possible correlations with histological changes and nerve density in herniated discs. This study also explores the presence of CEP in relation to VEP defects visible on MRI and identifies the predictive value of MRI in ascertaining the prognosis of the conservative management of herniated discs.

\section{Materials and Methods}

\section{Sample collection}

Fifty-one patients undergoing lumbar disc excision surgery for disc herniation in the orthopedic department of Ghurki Trust Teaching Hospital, Lahore, Pakistan were included in this study. Informed consent was obtained from the patients, and the study was approved by the Ethical Research Committee of University of Health Sciences, Lahore, Pakistan (IRB approval no., ERC/3789). Disc samples were collected and stored in $10 \%$ formalin immediately after surgery. Pre-surgical T1 and T2 weighted lumbar MRI and relevant demographic and clinical data were also obtained.

\section{Magnetic resonance imaging assessment}

RadiAnt Dicom software (Medixant, Poznań, Poland) was used to analyze the MRI scans, and the affected disc level was assessed to obtain disc degeneration grades by using the Pfirrmann grading system on the basis of signal intensity changes and height [12]. The discs were graded from I to V. Homogenous bright white discs were graded as grade I, and black discs with a collapsed disc space were graded as grade $\mathrm{V}$. The presence or absence of endplate defects was also noted and categorized as typical or atypical defects [13]. The presence and type of Modic changes [14], along with the type of herniation, were noted. Assessment of high-intensity zones (HIZ) in intervertebral discs was conducted, and the location of such zones was recorded [15].

\section{Histological assessment}

Sections measuring $5 \mu \mathrm{m}$ in thickness were stained with 
hematoxylin and eosin, Alcian blue-periodic acid-Schiff, and toluidine blue for histological degeneration scoring (HDS) and glycosaminoglycan (GAG) assessment respectively. HDS was scored from 0 to 15 according to the criteria of Weiler et al. [16]. Scoring was conducted on the basis of cell density, presence of tears and fissures, granular changes, and mucus degeneration. Inflammation was also scored on an ordinal scale from 0 (no inflammation) to 3 (severe inflammation). Toluidine blue-stained sections were scored for GAG loss on a scale of 0 (no loss) to 3 (severe loss).

\section{Immunohistochemical expression of protein gene product 9.5}

Protein gene product 9.5 (PGP 9.5) antibody was used to detect the presence of general nerves [17]. Paraffin sections measuring $3 \mu \mathrm{m}$ in thickness were oven-dried and then deparaffinized. Antigen retrieval was done with Tris/ EDTA (ethylenediaminetetraacetic acid) buffer. Sections were treated with peroxidase blocking reagent, and the primary antibody (anti-PGP 9.5 Ab, DAKO; Agilent Technologies, Santa Clara, CA, USA) was applied at a dilution of 1:50 for 1 hour. Ready-to-use anti-rabbit anti-mouse immunoglobulin G-dextran coupled with peroxidase molecules was used as a secondary antibody and applied for 30 minutes. DAB (3,3'-diaminobenzidine) chromogen was applied for 10 minutes. Sections were counterstained with hematoxylin solution for 30 seconds and then mounted with aqueous mounting medium. Positive nerve stains revealed brownish spots, and nerve density was recorded as spots $/ \mathrm{mm}^{2}$.

\section{Statistical analysis}

IBM SPSS ver. 24.0 (IBM Corp., Armonk, NY, USA) was used for statistical analysis. The data are presented as mean \pm standard deviation (SD) for numeric variables. Means between two groups were compared with independent-sample $t$-test, and three means were compared with one-way analysis of variance (ANOVA). The chisquare test was used for qualitative assessment. Two-way ANOVA was performed to assess whether any interaction between the two independent variables and the dependent variables exists. Correlations between continuous variables were determined using Pearson's product correlation. Statistical significance was established at $p<0.05$.

Table 1. Magnetic resonance imaging evaluation details of samples

\begin{tabular}{|c|c|c|c|c|c|c|c|}
\hline \multirow{2}{*}{ Variable } & \multirow{2}{*}{ Total disc samples $(n=51)$} & \multirow{2}{*}{ Age (yr) } & \multicolumn{2}{|c|}{ Gender } & \multirow{2}{*}{$\begin{array}{l}\mathrm{L} 3-\mathrm{L} 4 \\
(\mathrm{n}=2)\end{array}$} & \multirow{2}{*}{$\begin{array}{l}\mathrm{L}-\mathrm{L} 5 \\
(\mathrm{n}=29)\end{array}$} & \multirow{2}{*}{$\begin{array}{l}\mathrm{L5}-\mathrm{S} 1 \\
(\mathrm{n}=20)\end{array}$} \\
\hline & & & Male $(n=31)$ & Female $(n=20)$ & & & \\
\hline \multirow[t]{2}{*}{ Presence of endplate defects } & Yes $(n=30)$ & $38.5 \pm 11.0$ & 19 & 11 & 0 & 18 & 12 \\
\hline & No $(n=21)$ & $39.1 \pm 13.2$ & 12 & 9 & 2 & 11 & 8 \\
\hline \multirow[t]{3}{*}{ Types of herniation } & Protrusion $(n=10)$ & $37.3 \pm 9.8$ & 7 & 3 & 0 & 7 & 3 \\
\hline & Extrusion $(n=30)$ & $38.3 \pm 12.8$ & 16 & 14 & 2 & 19 & 9 \\
\hline & Sequestration $(n=11)$ & $41.6 \pm 13.4$ & 8 & 3 & 0 & 8 & 8 \\
\hline \multirow[t]{4}{*}{ Pfirrmann score } & II $(\mathrm{n}=3)$ & $24.3 \pm 5.5$ & 3 & 0 & 0 & 3 & 0 \\
\hline & III (n=26) & $40.1 \pm 11.4$ & 15 & 11 & 2 & 17 & 7 \\
\hline & IV $(n=20)$ & $39.25 \pm 13.6$ & 11 & 9 & 0 & 9 & 11 \\
\hline & $V(n=2)$ & $40.0 \pm 2.8$ & 2 & 0 & 0 & 0 & 2 \\
\hline \multirow[t]{2}{*}{ Modic changes } & Present $(n=16)$ & $40.1 \pm 12.8$ & 9 & 7 & 0 & 6 & $10^{\mathrm{a})}$ \\
\hline & Absent $(n=35)$ & $38.3 \pm 12.1$ & 22 & 13 & 2 & 23 & $10^{\mathrm{a})}$ \\
\hline \multirow[t]{2}{*}{ High-intensity zones } & Present $(n=6)$ & $40.2 \pm 15.2$ & 4 & 2 & 0 & 5 & 1 \\
\hline & Absent $(n=45)$ & $38.7 \pm 12.0$ & 27 & 18 & 2 & 24 & 19 \\
\hline
\end{tabular}

Values are presented as mean \pm standard deviation or number, unless otherwise stated.

a) Indicates borderline significance $(p=0.05)$. 


\section{Results}

\section{Demographic and MRI data}

The 51 disc samples analyzed in this study were obtained from spinal levels L3-S1. Among the discs obtained, $19.6 \%$ were protruded, $58.8 \%$ were extruded, and $21.6 \%$ were sequestered. The average age of the patients was $38.8 \pm 12.7$ years, and the male-to-female ratio was $31 / 20$. MRI observation showed that the Pfirrmann disc degeneration grades of the affected level ranged from II to V. VEP defects were present in nearly 59\% of the samples, among which approximately $37 \%$ were typical and nearly $16 \%$ showed both VEP affected. Modic changes were present in $31 \%$ of the samples, and all changes noted were of type II. Approximately $81 \%$ of the Modic changes were accompanied with defects. HIZ was observed in the posterior and anterior aspects of the discs in $12 \%$ of the samples. No significant difference in age, gender, and spinal level was observed across Pfirrmann grades, types of disc herniation, presence, and types of defects, and presence of HIZ. However, the lower lumbar region (L5-S1) was the region most affected $(p=0.05)$ by Modic changes (Table 1 ).
Significant associations between type of disc herniation and VEP defects were found, and all sequestered discs revealed VEP defects $(p=0.007)$. Pfirrmann scores were also significantly higher in sequestered discs $(p=0.001)$ than in other types of herniation.

\section{Histological findings}

The outer annulus fibrosus tissue was characterized by crimped collagen fibers organized into nearly parallel lamellae with alternating fiber angles and elongated fibroblast-like cells. The inner annulus fibrosus revealed an irregular arrangement of collagen fibers with relatively round, chondrocyte-like cells. Only two samples revealed nucleus pulposus tissues, which showed a homogenous matrix and round, chondrocyte-like cells. Distinguishing between the inner annulus and the nucleus pulposus was sometimes challenging because both tissues presented a uniform medium with few fibers and rounded cells. This was mainly due to the fibrosis of nucleus pulposus. Nearly $51 \%$ of the samples revealed CEP within the disc tissue manifesting as a glassy homogenous hyaline structure.

Histological assessment of the sections revealed HDS
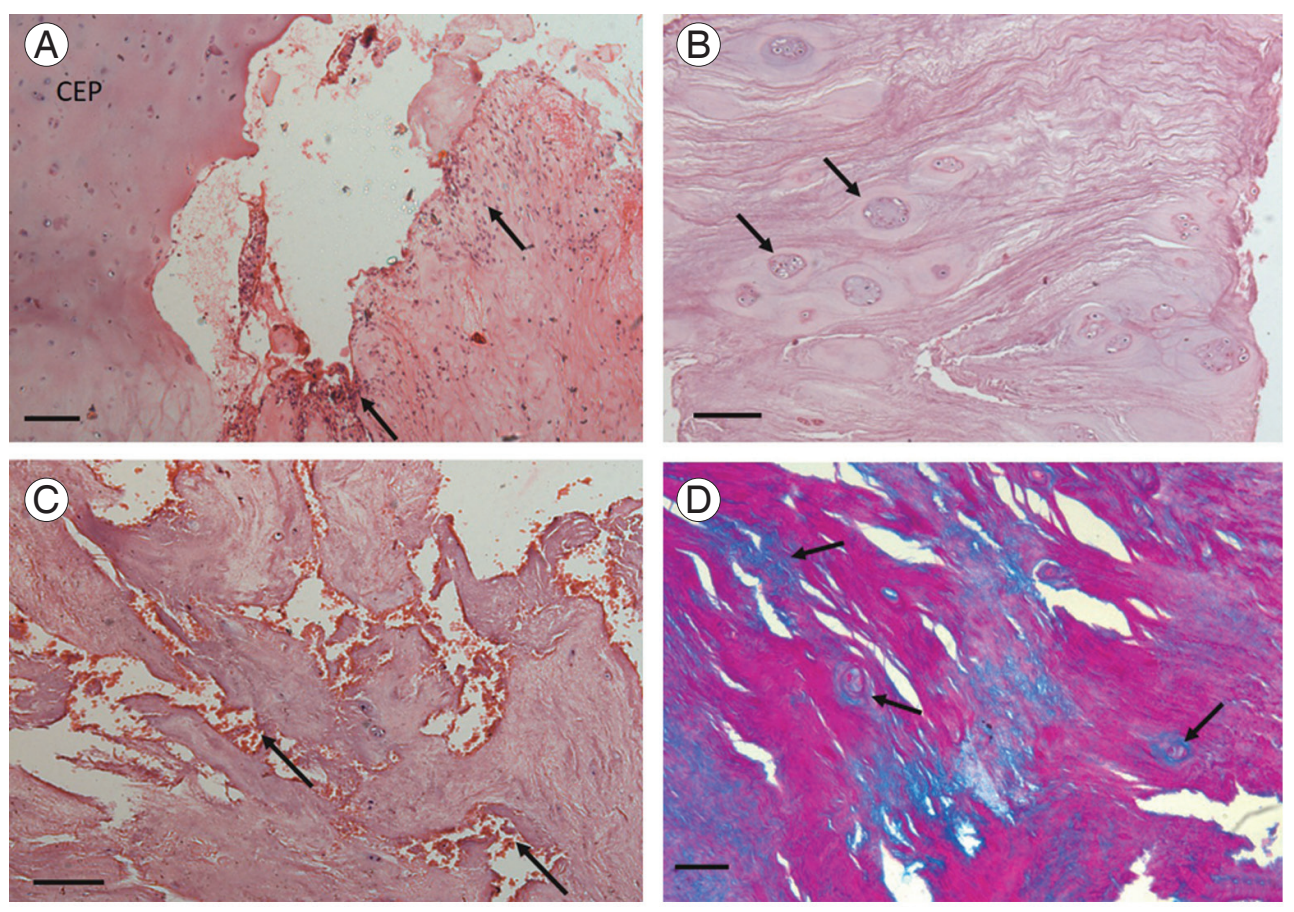

Fig. 1. Images showing histological features of herniated discs. (A) Presence of cartilaginous endplate (CEP) and adjacent inflammatory infiltrate (black arrows) (H\&E stain, $\times 200 \mu \mathrm{m}$ ). (B) Increase in cell density with moderate sized cell clusters (black arrows) (H\&E stain, $\times 200 \mu \mathrm{m})$. (C) Occurrence of granular changes and structural alterations (black arrows) (H\&E stain, $\times 200$ $\mu \mathrm{m})$. (D) Mucus degeneration indicated by blue stain (black arrows) (Alcian blue-periodic acid-Schiff stain, $\times 100 \mu \mathrm{m}$ ). 
of $2-12$, with a mean $\pm \mathrm{SD}$ of $6.8 \pm 2.5$. Nearly $88 \%$ of the sections showed inflammation; among the sections analyzed, $36 \%$ showed mild inflammation, $42 \%$ had moderate inflammation, and $22 \%$ had severe inflammation (Fig. 1). GAG loss was observed in all sections except one, and the grading scores indicated that $33 \%$ of the samples had mild loss, $41 \%$ had moderate loss, and $24 \%$ had severe loss.

No significant effect of age, gender, and spinal level on HDS and inflammation scores was noted, but GAG loss was significantly higher in males $(p=0.002)$ than in females. Age showed a slight influence $(p=0.08)$ on the presence of CEP (Table 2).

\section{Association between histological and magnetic reso- nance imaging parameters}

Discs with adjacent endplate defects showed an overall increase in HDS $(p<0.001)$; all individual variables of HDS, including cell density $(p=0.02)$, structural alterations $(p<0.001)$, granular changes $(p=0.002)$, and mucus degeneration ( $p=0.001$ ), also significantly increased (Fig. 2). Inflammation scores were higher in disc tissues with adjacent VEP defects $(p<0.001)$ than in those without. The type of defect and number of VEPs affected did not reveal a significant difference in terms of any parameter of HDS,

Table 2. Histological evaluation details of samples

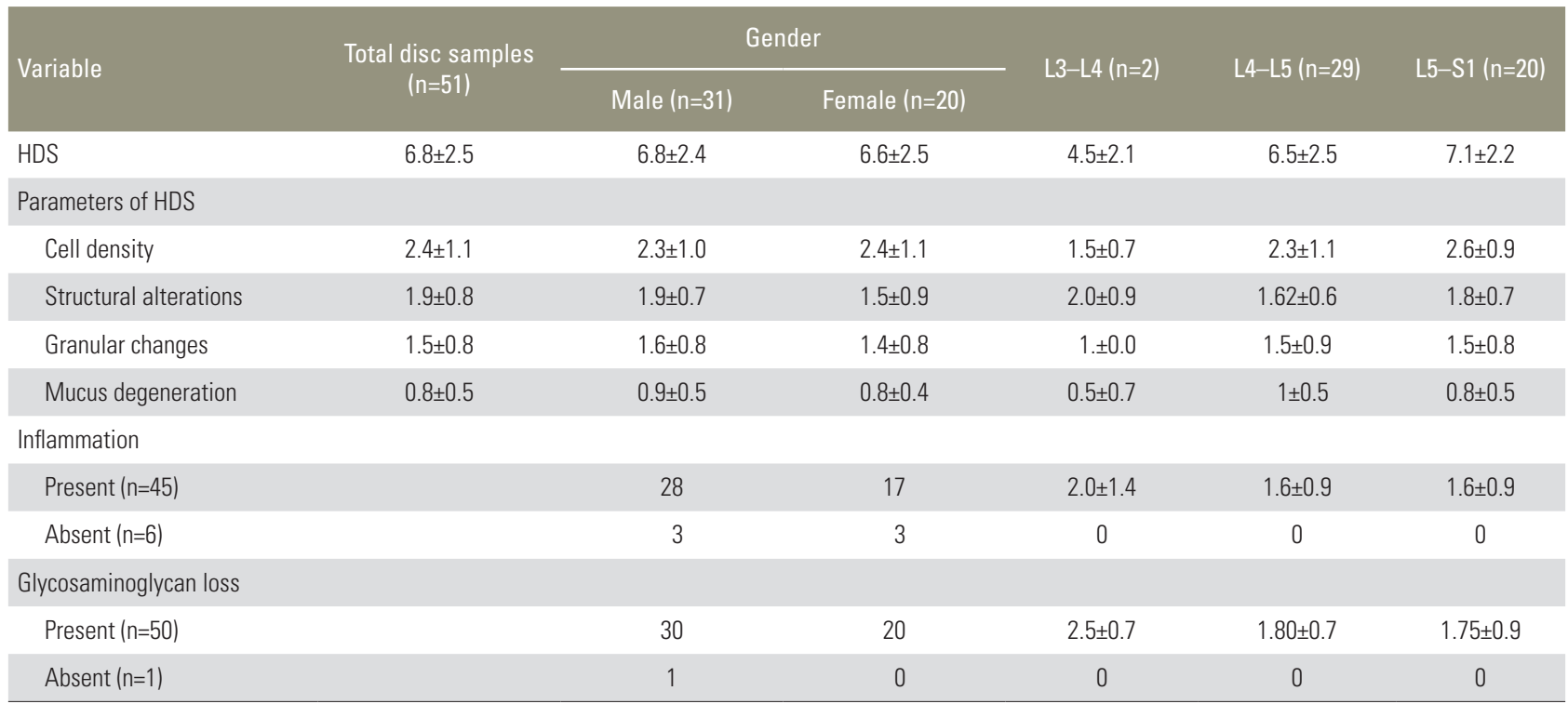

Values are presented as mean \pm standard deviation or number, unless otherwise stated. HDS, histological degeneration scoring.
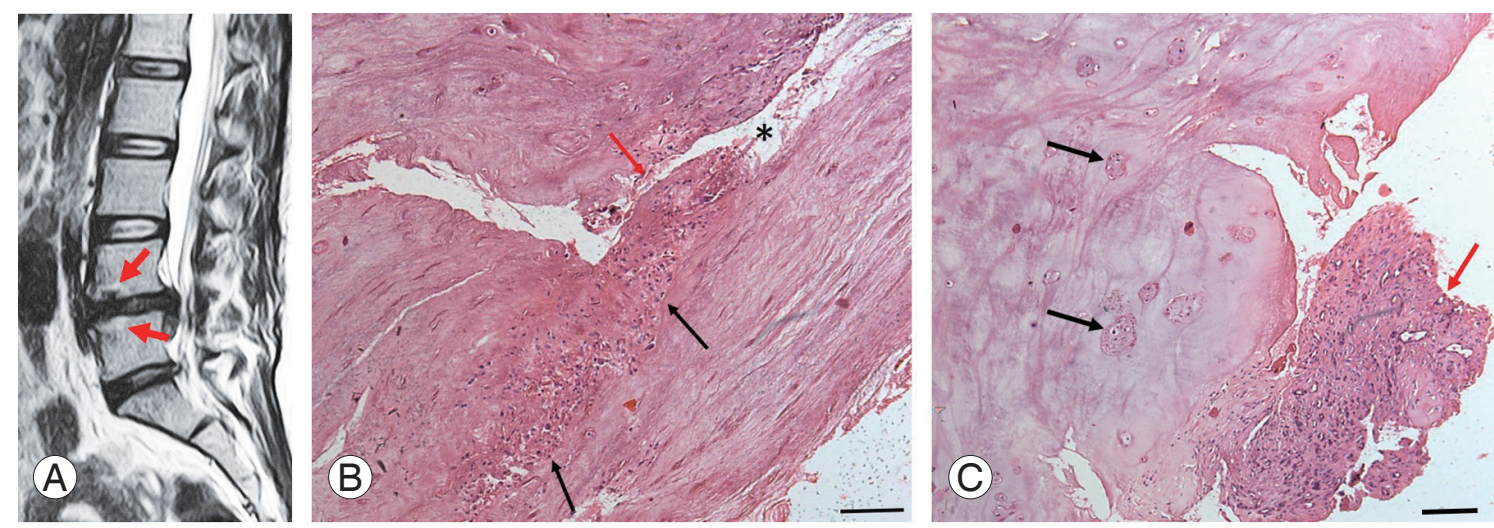

Fig. 2. Representative image of a 45-year-old female. (A) T2 weighted lumbar sagittal magnetic resonance imaging showing vertebral endplate defects with Modic changes (red arrows). (B) H\&E-stained section showing inflammatory infiltrate (black arrows), structural alteration (asterisk), and granulation tissue (red arrow) (H\&E stain, $\times 200 \mu \mathrm{m})$. (C) H\&E-stained section showing small to medium sized cell clusters (black arrows) and inflammatory infiltrate (red arrow) (H\&E stain, $\times 200 \mu \mathrm{m})$. 
Table 3. Association between MRI \& histological parameters

\begin{tabular}{|c|c|c|c|c|c|c|c|}
\hline MRI parameters & Cell density & Tears & Granular changes & Mucus degeneration & HDS & Inflammation & GAG loss \\
\hline \multicolumn{8}{|l|}{ End plate defects } \\
\hline Present & $2.7 \pm 1.1^{* *}$ & $2.2 \pm 0.8^{* * *}$ & $1.8 \pm 0.7^{* *}$ & $1.1 \pm 0.4^{* *}$ & $7.8 \pm 2^{* * *}$ & $2.03 \pm 0.8^{* * *}$ & $2.0 \pm 0.8$ \\
\hline Absent & $2.0 \pm 0.8$ & $1.4 \pm 0.5$ & $1.1 \pm 0.8$ & $0.5 \pm 0.5$ & $5.1 \pm 1.9$ & $1.1 \pm 0.7$ & $1.6 \pm 0.7$ \\
\hline \multicolumn{8}{|l|}{ Types of herniation } \\
\hline Protrusion $(n=10)$ & $1.6 \pm 0.5^{*}$ & $1.6 \pm 0.6^{*}$ & $1.2 \pm 0.6$ & $0.8 \pm 0.4$ & $5.2 \pm 1.4^{*}$ & $1.2 \pm 0.9$ & $1.9 \pm 0.8$ \\
\hline Extrusion ( $n=30)$ & $2.5 \pm 1.0$ & $1.8 \pm 0.7$ & $1.5 \pm 0.9$ & $0.8 \pm 0.6$ & $6.7 \pm 2.3$ & $1.7 \pm 0.8$ & $1.8 \pm 0.7$ \\
\hline Sequestration $(n=11)$ & $2.8 \pm 1.3$ & $2.4 \pm 1.0$ & $1.8 \pm 0.7$ & $1.2 \pm 0.4$ & $8.3 \pm 2.4$ & $1.8 \pm 1.1$ & $2.0 \pm 1.0$ \\
\hline \multicolumn{8}{|l|}{ Modic changes } \\
\hline Present & $3.1 \pm 1.0^{* *}$ & $2.1 \pm 0.8$ & $1.7 \pm 0.7$ & $1.0 \pm 0.3$ & $8.0 \pm 2.1^{*}$ & $1.9 \pm 0.9$ & $2.0 \pm 0.7$ \\
\hline Absent & $2.0 \pm 0.9$ & $1.8 \pm 0.7$ & $1.4 \pm 0.8$ & $0.8 \pm 0.6$ & $6.1 \pm 2.3$ & $1.5 \pm 0.7$ & $1.7 \pm 0.8$ \\
\hline \multicolumn{8}{|l|}{ HIZ } \\
\hline Present & $2.6 \pm 1.5$ & $2.5 \pm 0.8$ & $2.0 \pm 1.2^{*}$ & $1.0 \pm 0.8^{*}$ & $8.1 \pm 3.8^{* *}$ & $2.1 \pm 0.9^{*}$ & $1.8 \pm 0.8$ \\
\hline Absent & $2.3 \pm 0.9$ & $1.8 \pm 0.8$ & $1.4 \pm 0.7$ & $0.8 \pm 0.5$ & $6.5 \pm 2.1$ & $1.5 \pm 0.9$ & $1.6 \pm 0.5$ \\
\hline \multicolumn{8}{|l|}{ Pfirrmann grades } \\
\hline$\|$ & $1.6 \pm 0.5$ & $1.6 \pm 0.5$ & $1.0 \pm 0.0$ & $0.3 \pm 0.5$ & $4.6 \pm 0.5$ & $1.0 \pm 1.0$ & $2.6 \pm 0.5$ \\
\hline III & $2.1 \pm 0.9$ & $1.8 \pm 0.8$ & $1.5 \pm 0.9$ & $0.5 \pm 0.5$ & $6.5 \pm 2.3$ & $1.7 \pm 0.8$ & $1.8 \pm 0.7$ \\
\hline IV & $2.7 \pm 1.1$ & $1.9 \pm 0.8$ & $1.5 \pm 0.7$ & $0.9 \pm 0.6$ & $7.1 \pm 2.5$ & $1.5 \pm 0.9$ & $1.7 \pm 0.8$ \\
\hline V & $3.0 \pm 1.4$ & $2.5 \pm 0.7$ & $2.0 \pm 1.4$ & $1.0 \pm 0.0$ & $8.5 \pm 3.5$ & $2.5 \pm 0.7$ & $2.0 \pm 0.0$ \\
\hline
\end{tabular}

Values are presented as mean \pm standard deviation.

$\mathrm{MRI}$, magnetic resonance imaging; $\mathrm{HIZ}$, high-intensity zones.

${ }^{*} p<0.05 .{ }^{* *} p<0.01 .{ }^{* * *} p<0.001$.

HDS, or inflammation (Table 3).

Discs with adjacent Modic changes showed increased overall HDS ( $p=0.01)$, especially cell density $(p=0.001)$. Discs with HIZ also revealed increased HDS $(p=0.003)$, along with the individual variables of HDS granular changes $(p=0.01)$ and mucus degeneration $(p=0.05)$, inflammation is also seen to be increased in these discs with borderline significance $(p=0.05)$.

The types of herniation showed a significant effect on HDS and some of its features. Extruded and sequestered discs showed increased cell density $(p=0.02)$, structural alterations $(p=0.03)$, and HDS $(p=0.01)$ compared with protruded discs.

Approximately $66 \%$ of the population with visible MRI defects revealed CEPs in their herniated tissue. CEPs were also found in nearly $28 \%$ of those individuals without visible VEP defects on MRI. Histological sections with CEP showed higher overall HDS $(p<0.001)$ and inflammation $(p<0.001)$; all individual variables of HDS, including cell density ( $p=0.001)$, structural alterations $(p<0.001)$, granular changes $(p=0.006)$, and mucus degeneration $(p=0.001)$, also significantly increased. Modic changes were signifi- cantly higher at levels where disc tissues included a CEP $(p=0.02)$. The presence of CEP was not affected by the type of disc herniation.

Simple main effect analysis showed that HDS scores were significantly higher in samples with defects $(p=0.003)$ and CEP $(p=0.008)$ as compared to Modic changes. However, a borderline significant interaction between the effects of Modic changes and the presence of CEP on inflammation scores was observed $(F=3.76, p=0.05)$.

\section{Immunohistochemical expression of protein gene product 9.5}

The immunohistochemical expression of PGP 9.5 was evident in 50/51 samples (Fig. 3). The mean nerve density was $0.020 \pm 0.015 / \mathrm{mm} 2$. No nerve was found in the CEP of the disc tissue. Nerve density was significantly correlated with cell density ( $r=0.29, p=0.01$ ), mucus degeneration $(r=0.27, p=0.02)$, HDS $(r=0.27, p=0.02)$, and inflammation $(r=0.2, p=0.08)$. However, no MRI parameter showed a statistical association with nerve density. 

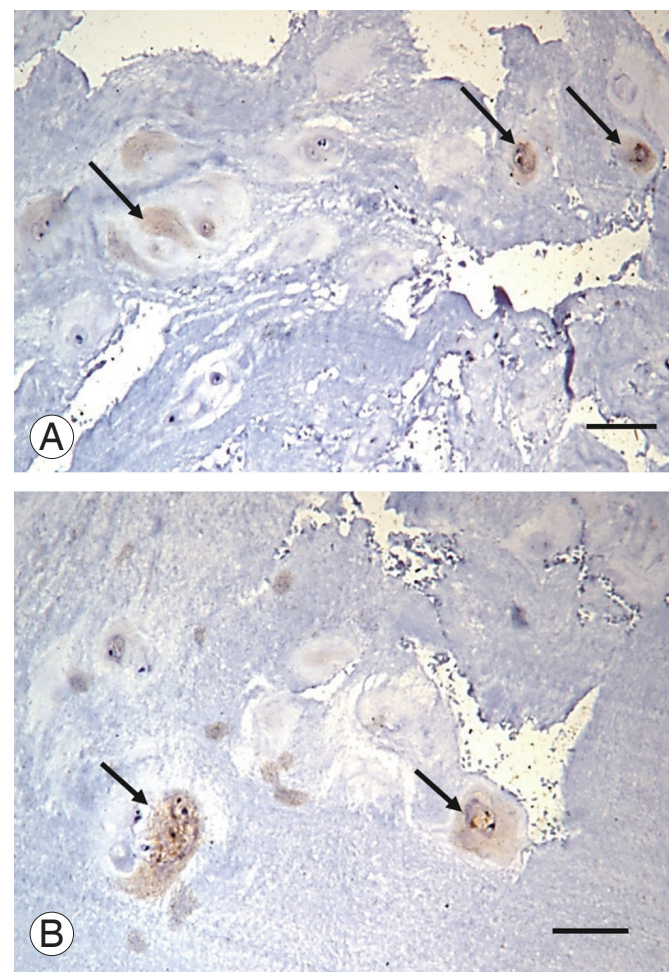

Fig. 3. (A, B) Immunohistochemical expression of protein gene product 9.5 (black arrows) in tissue sections of 48-year-old male (×200 $\mu \mathrm{m})$.

\section{Discussion}

Knowledge of the histological composition of herniated intervertebral discs is important to evaluate their role in regression, pain, and other clinical symptoms. To the best of our knowledge, this study is the first to associate the histological features of herniated disc with VEP changes and other MRI parameters. The findings revealed strong associations among VEP defects, Modic changes, types of herniation, and HIZ with disc degeneration and inflammation. Over half of the samples exhibited CEP, and the presence of this feature correlated well with the VEP defects observed on MRI and the Modic changes noted. This finding indicates that CEP may be a reliable tool to assess the histological composition of herniated discs.

The loose connection between cartilage and bony endplates has been postulated to explain why the mechanical pull of the annulus fibers can strip the CEP from the bone [11]. In the current study, this finding can be explained from two perspectives. First, the mechanical pull during disc herniation is sufficiently strong to create a bony defect at the same level while stripping the CEP. Second, pre-existing defects in the VEP may promote cartilage avulsions [18], which are easily pulled off during disc her- niation. In both scenarios, a free portal through which cytokines, inflammatory cells, and bacteria are able to move from the bone marrow to the nucleus and vice versa may be established [11], which could explain the strong association between the presence of CEP and the high HDS and inflammatory scores noted in the current study. CEP fragments are also known to express the pro-inflammatory cytokine tumor necrosis factor- $\alpha$ (TNF- $\alpha$ ) in herniated disc tissues [11], thus supporting the finding of increased HDS and inflammation in the current study.

The presence of CEP in the disc tissue has been linked to the slow resorption of herniated discs and persistence of symptoms. A previous study reported a negative correlation between the presence of CEP in the herniated disc and the inflammatory response, eventually concluding that the failure of the discs to resorb, which results in the poor remission of clinical symptoms, may be due to reductions in the inflammatory response [19]; however, this finding contradicts our results. Regression of the herniated disc and clinical symptoms do not always appear to be correlated [8], which means compression may not be the only reason behind the persistence of clinical symptoms; the release of inflammatory cytokines and the composition of the herniated disc itself may also contribute to the phenomena observed $[10,20]$.

While the role of inflammation during the regression of herniated intervertebral discs has been well acknowledged [21], the inflammatory reaction may also cause adhesions between the lesion and nerve root, leading to severe tension or compression on the latter [21-23]. The production of inflammatory cytokines has been suggested to be a significant contributor to the manifestation of pain [21] by inducing the ingrowth of nerves and blood vessels [24]. The significant association between nerve density and increased degeneration and inflammation scores observed in the current study supports this belief. We were unable to assess the expression of inflammatory cytokines and sensory nerve markers in the current study, which is a major limitation of this work. However, the presence of CEP may be assumed to indicate barrier loss and participate in the production of inflammatory cytokines, such as TNF- $\alpha$; in this case, inflammation, instead of helping in regression, may hinder sufficient clinical improvement.

\section{Conclusions}

In conclusion, VEP changes can modify the degeneration 
and inflammation scores of herniated discs at the same level. The presence of these changes is highly predictive of the occurrence of CEP in the prolapsed disc, which may lead to slow resorption and the persistence of clinical symptoms owing to the release of inflammatory cytokines either directly from the CEP or due to barrier loss.

\section{Conflict of Interest}

No potential conflict of interest relevant to this article was reported.

\section{Acknowledgments}

This work was supported by University of Health Sciences, Lahore, Pakistan.

\section{References}

1. Deyo RA, Loeser JD, Bigos SJ. Herniated lumbar intervertebral disk. Ann Intern Med 1990;112:598-603.

2. Adams MA, McNally DS, Chinn H, Dolan P. The clinical biomechanics award paper 1993 Posture and the compressive strength of the lumbar spine. Clin Biomech (Bristol, Avon) 1994;9:5-14.

3. Dydyk AM, Ngnitewe Massa R, Mesfin FB. Disc herniation. Treasure Island (FL): StatPearls Publishing; 2020.

4. Gugliotta M, da Costa BR, Dabis E, et al. Surgical versus conservative treatment for lumbar disc herniation: a prospective cohort study. BMJ Open 2016;6:e012938.

5. Schoenfeld AJ, Weiner BK. Treatment of lumbar disc herniation: evidence-based practice. Int J Gen Med 2010;3:209-14.

6. Yao M, Xu BP, Li ZJ, et al. A comparison between the low back pain scales for patients with lumbar disc herniation: validity, reliability, and responsiveness. Health Qual Life Outcomes 2020;18:175.

7. Chiu CC, Chuang TY, Chang KH, Wu CH, Lin PW, Hsu WY. The probability of spontaneous regression of lumbar herniated disc: a systematic review. Clin Rehabil 2015;29:184-95.

8. Turk O, Antar V, Yaldiz C. Spontaneous regression of herniated nucleus pulposus: the clinical findings of 76 patients. Medicine (Baltimore) 2019;98:e14667.

9. Benson RT, Tavares SP, Robertson SC, Sharp R, Mar- shall RW. Conservatively treated massive prolapsed discs: a 7-year follow-up. Ann R Coll Surg Engl 2010;92:147-53.

10. Willburger RE, Ehiosun UK, Kuhnen C, Kramer J, Schmid G. Clinical symptoms in lumbar disc herniations and their correlation to the histological composition of the extruded disc material. Spine (Phila Pa 1976) 2004;29:1655-61.

11. Lama P, Zehra U, Balkovec C, et al. Significance of cartilage endplate within herniated disc tissue. Eur Spine J 2014;23:1869-77.

12. Pfirrmann CW, Metzdorf A, Zanetti M, Hodler J, Boos N. Magnetic resonance classification of lumbar intervertebral disc degeneration. Spine (Phila $\mathrm{Pa}$ 1976) 2001;26:1873-8.

13. Samartzis D, Mok FP, Karppinen J, Fong DY, Luk KD, Cheung KM. Classification of Schmorl's nodes of the lumbar spine and association with disc degeneration: a large-scale population-based MRI study. Osteoarthritis Cartilage 2016;24:1753-60.

14. Modic MT. Modic type 1 and type 2 changes. J Neurosurg Spine 2007;6:150-1.

15. Aprill C, Bogduk N. High-intensity zone: a diagnostic sign of painful lumbar disc on magnetic resonance imaging. Br J Radiol 1992;65:361-9.

16. Weiler C, Lopez-Ramos M, Mayer HM, et al. Histological analysis of surgical lumbar intervertebral disc tissue provides evidence for an association between disc degeneration and increased body mass index. BMC Res Notes 2011;4:497.

17. Lama P, Le Maitre CL, Harding IJ, Dolan P, Adams MA. Nerves and blood vessels in degenerated intervertebral discs are confined to physically disrupted tissue. J Anat 2018;233:86-97.

18. Fields AJ, Rodriguez D, Gary KN, Liebenberg EC, Lotz JC. Influence of biochemical composition on endplate cartilage tensile properties in the human lumbar spine. J Orthop Res 2014;32:245-52.

19. Kawaguchi K, Harimaya K, Matsumoto Y, et al. Effect of cartilaginous endplates on extruded disc resorption in lumbar disc herniation. PLoS One 2018;13:e0195946.

20. Siddall PJ, Cousins MJ. Spinal pain mechanisms. Spine (Phila Pa 1976) 1997;22:98-104.

21. Kobayashi S, Meir A, Kokubo Y, et al. Ultrastructural analysis on lumbar disc herniation using surgical specimens: role of neovascularization and macro- 
phages in hernias. Spine (Phila Pa 1976) 2009;34:65562.

22. Rydevik B, Brown MD, Lundborg G. Pathoanatomy and pathophysiology of nerve root compression. Spine (Phila Pa 1976) 1984;9:7-15.

23. Kobayashi S, Baba H, Uchida K, et al. Effect of mechanical compression on the lumbar nerve root: localization and changes of intraradicular inflammatory cytokines, nitric oxide, and cyclooxygenase. Spine (Phila Pa 1976) 2005;30:1699-705.

24. Ohtori S, Miyagi M, Inoue G. Sensory nerve ingrowth, cytokines, and instability of discogenic low back pain: a review. Spine Surg Relat Res 2018;2:11-7. 